INPLASY

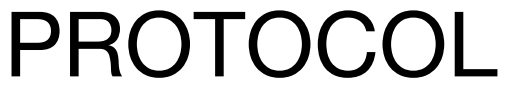

To cite: Xu et al. Evidencebased Acupuncture for Major Depressive Disorder: a

Systematic Review and MetaAnalysis of high-quality RCTs. Inplasy protocol 2021100073. doi:

10.37766/inplasy2021.10.0073

Received: 19 October 2021

Published: 19 October 2021

Corresponding author:

Guixing Xu

1032159472@qq.com

Author Affiliation:

Chengdu University of Traditional Chinese Medicine chengdu.

Support: National Natural Science Found.

Review Stage at time of this submission: Data analysis.

Conflicts of interest:

None declared.

\section{Evidence-based Acupuncture for Major Depressive Disorder: a Systematic Review and Meta-Analysis of high- quality RCTs}

Xu, GX1; Xiao, GW2; Huang, BQ33; Lei, HZ4; Yin, ZH5; Huang, LY6; Zhou, Z7; Tian, H8; Huang, FY9; Liu, YL10; Sun, MS11; Zhao, L'12; Liang, FR ${ }^{13}$.

Review question / Objective: Acupuncture has shown benefits in treating major depressive disorder (MDD) during clinical practice; however, the available evidence in the medical literature is contradictory.

Information sources: The following online databases were searched: EMBASE, The Cochrane Library, PubMed, The Cochrane Central Register of Controlled Trials (CENTRAL), Web of Science, The China National Knowledge Infrastructure (CNKI), The Wanfang database, The VIP Database for Chinese Technical Periodicals, The China Doctoral Dissertations Fulltext Database (CDFD). All RCTs were published from inception to 10th September 2021.

INPLASY registration number: This protocol was registered with the International Platform of Registered Systematic Review and Meta-Analysis Protocols (INPLASY) on 19 October 2021 and was last updated on 19 October 2021 (registration number INPLASY2021100073).

\section{INTRODUCTION}

Review question / Objective: Acupuncture has shown benefits in treating major depressive disorder (MDD) during clinical practice; however, the available evidence in the medical literature is contradictory.

Condition being studied: Major depressive disorder (MDD) is a common psychological condition with an estimated lifetime prevalence of $16 \%$, affecting more than 320 million people across the globe. As of April 2017, depression has been recognized by the World Health Organization (WHO) as the leading cause of health-related disability, accounting for approximately $4.4 \%$ of all disabilities and premature deaths worldwide. Antidepressants such as selective serotonin reuptake inhibitors or 
selective serotonin-norepinephrine reuptake inhibitors are commonly used to treat MDD. Most evidence-based guidelines recommend antidepressants to be the first-line therapy because of their favorable outcomes and superior characteristics, including broad-spectrum effectiveness, safety, tolerability, simplicity of use, and low cost. Nevertheless, pharmacological interventions also have undesirable side effects, including central nervous system and gastrointestinal disorders, weight gain, sexual dysfunction, and adverse emotional effects. In addition, long-term use may also cause drug tolerance, withdrawal symptoms when discontinued and increased suicidal ideation in certain patient populations. As an essential component of traditional Chinese medicine, acupuncture therapy has been practiced in China for thousands of years in disease prevention and treatment, functional improvement, longevity enhancement, and regulating emotions. As early as 1979, the WHO held a symposium on Acupuncture and created a list of 43 diseases suitable for Acupuncture6. In 2002, the WHO recommended Acupuncture as a treatment for depression (including depressive neurosis and depression following stroke). However, significant heterogeneity has been reported in findings of several randomized control trials (RCTs).

\section{METHODS}

Participant or population: Patients (aged $\geq$ 18 years) were diagnosed with MDD according to the third edition of Chinese Classification of Mental Disorders (CCMD-2/3), the International Statistical Classification of Diseases and Related Health Problems (ICD-10/11), or the Diagnostic and Statistical Manual of Mental Disorders (DSM-II/III/III-R/IV/IV-TR/V).

Intervention: The experimental group consisted of patients that received Acupuncture or Acupuncture plus antidepressants. The acupoint numbers, retaining time and frequency, treatment sessions were not limited.The experimental group consisted of patients that received
Acupuncture or Acupuncture plus antidepressants. The acupoint numbers, retaining time and frequency, treatment sessions were not limited. The control group included non-acupuncture techniques, such as placebo control or other active therapies.

Comparator: The control group included non-acupuncture techniques, such as placebo control or other active therapies.

Study designs to be included: We included all RCTs with eligible intervention(s) and outcome(s) for MDD published in Chinese and English. For the crossover trials with the randomized control design, the first stage consisted of data collection for this meta-analysis. Furthermore, we only included RCTs with a Jadad score $\geq 4$ since the previously published systematic review could not obtain accurate conclusions due to the low quality of RCTs included.

Eligibility criteria: Selection criteriaStudy designWe included all RCTs with eligible intervention(s) and outcome(s) for MDD published in Chinese and English. For the crossover trials with the randomized control design, the first stage consisted of data collection for this meta-analysis. Furthermore, we only included RCTs with a Jadad score $\geq 4$ since the previously published systematic review could not obtain accurate conclusions due to the low quality of RCTs included. Population Patients (aged $\geq 18$ years) were diagnosed with MDD according to the third edition of Chinese Classification of Mental Disorders (CCMD-2/3), the International Statistical Classification of Diseases and Related Health Problems (ICD-10/11), or the Diagnostic and Statistical Manual of Mental Disorders (DSM-II/III/III-R/IV/IV-TR/V). Interventions / C omparators The experimental group consisted of patients that received Acupuncture or Acupuncture plus antidepressants. The acupoint numbers, retaining time and frequency, treatment sessions were not limited.The control group included non-acupuncture techniques, such as placebo control or other active therapies.Outcome measuresthe primary outcome was the 
Hamilton Depression Rating Scale for Depression (HAMD17/24). The secondary outcomes included changes in the Zung Self-rating Depression Scale (SDS) and the incidence of adverse effects. The data at each time point was extracted from the original trials to be analyzed in this study.

Information sources: The following online databases were searched: EMBASE, The Cochrane Library, PubMed, The Cochrane Central Register of Controlled Trials (CENTRAL), Web of Science, The China National Knowledge Infrastructure (CNKI), The Wanfang database, The VIP Database for Chinese Technical Periodicals, The China Doctoral Dissertations Full-text Database (CDFD). All RCTs were published from inception to 10th September 2021.

Main outcome(s): The primary outcome was the Hamilton Depression Rating Scale for Depression (HAMD17/24).

Additional outcome(s): The secondary outcomes included changes in the Zung Self-rating Depression Scale (SDS) and the incidence of adverse effects.

Quality assessment / Risk of bias analysis: The risk of bias of eligible trials was measured by the Risk of Bias (ROB) Tool in Cochrane Handbook (5.1.0) by two independent researchers (HFY and LYL). Each criterion was graded as "low" risk of bias, "high" risk of bias and "unclear" risk of bias. The methodology quality was assessed by two independent investigators (XQW and HBQ) with the Jadad scale. Any dissent occured in the assessment procedures was judged by a third investigator (SMS).

Strategy of data synthesis: Meta-analysis of RCTs with available data was performed by calculating the effect size and $95 \% \mathrm{CI}$ using the random-effects model. Heterogeneity among trials was identified by the $\mathrm{X} 2$ test and reported as 12 . Statistical analyses were performed with RevMan 5.2 and Stata 15.0. Two-sided P-value $<0.05$ was considered statistically significant. Studies were grouped according to the type of intervention (acupuncture,
Acupuncture plus antidepressants) and the controls (sham-acupuncture, antidepressants, and sham-acupuncture plus antidepressants). For studies with more than 1 control group, such as Acupuncture versus sham acupuncture versus antidepressants, the results were split into pairwise comparisons by the different comparators.

Subgroup analysis: Meta-regression and sensitivity analyses were conducted to explore potential sources of heterogeneity. Meta-regression was used to explore whether the age of patients, baseline HAMD-24, course of MDD, and acupuncture session affect the effectiveness of Acupuncture. Sensitivity analysis was used to identify studies that significantly affected the overall effect.

Sensitivity analysis: Meta-regression and sensitivity analyses were conducted to explore potential sources of heterogeneity. Meta-regression was used to explore whether the age of patients, baseline HAMD-24, course of MDD, and acupuncture session affect the effectiveness of Acupuncture. Sensitivity analysis was used to identify studies that significantly affected the overall effect.

Country(ies) involved: China.

Keywords: Acupuncture, depression, metaanalysis.

Contributions of each author:

Author 1 - Guixing Xu.

Author 2 - Qiwei Xiao.

Author 3 - Biqing Huang.

Author 4 - Hanzhou Lei.

Author 5 - Zihan Yin.

Author 6 - Liuyang Huang.

Author 7 - Zhuo Zhou.

Author 8 - Hao Tian.

Author 9 - Fengyuan Huang.

Author 10 - Yilin Liu.

Author 11 - Mingsheng Sun.

Author 12 - Ling Zhao.

Author 13 - Fanrong Liang. 